\title{
NOVO INDICADOR COINCIDENTE PARA A ATIVIDADE INDUSTRIAL BRASILEIRA
}

\author{
Gilberto Hollauer ${ }^{\S}$ \\ João Victor Issler ${ }^{\alpha}$ \\ Hilton H. Notini ${ }^{\dagger}$
}

\section{RESUMO}

Neste trabalho aplicam-se e testam-se, dentro da amostra, algumas metodologias de construção de indicadores coincidentes para atividade industrial visando à detecção de ciclos de crescimento/recessão da atividade industrial. Especificamente, testou-se uma versão baseada no indicador coincidente do The Conference Board (TCB), uma versão modificada deste, na qual as volatilidades são modeladas, a abordagem de Stock-Watson tradicional e, finalmente, testou-se a abordagem de Mariano-Murasawa.

Concluiu-se que, em geral, o índice TCB padrão é superior a outros métodos testados, sendo apenas competitivo com o método modificado. Mais ainda, o índice TCB tende a englobar os períodos recessivos apresentados pelos outros índices, sendo correspondentes com os períodos recessivos conhecidos do setor industrial.

Palavras-chave: Modelos de Séries Temporais, indicadores coincidentes, ciclos de negócios.

\section{ABSTRACT}

In this paper we perform and evaluate, in sample, some methodologies for building of coincident indicators focusing on the detection of business cycle of the Industrial activity. Specifically, we try a version of coincident indicator based on the coincident indicator for economy of the TCB (The Conference Board), a modified version in which the volatility is modeled, a Stock-Watson approach and a Mariano-Murasawa approach.

We conclude that, in general, the TCB index out-performs the others methods tested, being competitive just with the modified method. Furthermore, The TCB Traditional has the tendency to comprehend the recessives periods of the others, in correspondence with the known recessive periods for industrial activity.

Keywords: Time Series Models, coincident index, business cycles.

JEL classification: C32, E32.

$\S \quad$ Ministério das Minas e Energia. Endereço para contato: Esplanada dos Ministérios Bloco "U". CEP: 70.065-900 - Brasília - DF. Email: gilberto.hollauer@mme.gov.br.

a Fundação Getulio Vargas, Escola de Pós-Graduação em Economia. Endereço para contato: P. Botafogo, 190 , s. 1100 - Rio de Janeiro - RJ. CEP: 22250-900. E-mail: joao.issler@fgv.br.

$\dagger$ Fundação Getulio Vargas, Escola de Pós-Graduação em Economia e Agência Nacional de Aviação Civil. Endereço para contato: Av. Presidente Vargas, 850 - Rio de Janeiro - RJ. CEP 20210-030. Email: hilton@fgvmail.br

Recebido em novembro de 2007. Aceito para publicação em setembro de 2008. 


\section{INTRODUÇÃO}

Toda sociedade tem interesse em saber qual é o estado atual da economia (recessão ou expansão). Porém, o estado da economia é não observável e não há consenso sobre a melhor forma de estimar essa variável latente. A inexistência de uma medida direta do estado da economia levou a construções de proxies deste que possam ser usadas em tempo real - são os chamados indicadores coincidentes da atividade econômica.

O estudo dos indicadores coincidentes auxilia na compreensão das flutuações econômicas, sendo importante tanto para o setor público, na formulação e execução de políticas públicas, quanto para planejamento do setor privado.

Nos EUA, as pesquisas sobre os ciclos de negócios iniciaram-se há aproximadamente um século pelo National Bureau of Economic Research - NBER-, de forma que hoje a literatura aplicada àquele país já se encontra bastante desenvolvida e consolidada. ${ }^{1}$ Naquele país, hoje coexistem métodos heurísticos e estatístico-econométricos de construção de indicadores coincidentes e antecedentes.

No Brasil, com a exceção de Contador (1977), o assunto somente despertou atenção após o fim de nosso último período inflacionário, mais precisamente, somente após o ano $2000 .{ }^{2}$ Dessa forma, os estudos aplicados brasileiros ainda são relativamente escassos e a avaliação das diferentes técnicas por experiências repetidas ainda não é tão consolidada como a experiência americana.

Uma informação frequentemente desprezada na confecção de indicadores coincidentes é a informação trimestral disponível, o que pode ser extremamente ineficiente, já que várias séries que apresentam alta correlação com o estado da economia não se encontram disponíveis em frequência mensal. Um bom exemplo é o Produto Interno Bruto (PIB) que, no Brasil, como na maioria dos países, é medido em frequência trimestral.

Ocorre que a composição de indicadores a partir de séries de periodicidades diferentes é estatisticamente complicada. Mariano e Murasawa (2003) ofereceram uma metodologia que agrega essa informação, tratando a variável trimestral como uma variável latente observada somente a cada três meses.

No presente trabalho, seguindo a metodologia de Mariano e Murasawa (2003), nós construímos um indicador coincidente para a atividade industrial brasileira baseado em séries de dupla periodicidade, visando, a partir deste, a detecção de ciclos de crescimento/recessão da atividade industrial. Além disso, construímos mais três indicadores coincidentes para a atividade industrial: uma versão baseada no indicador coincidente do The Conference Board (TCB), uma versão modificada deste, na qual as volatilidades são modeladas, e uma baseada na abordagem

1 Ver Burns e Mitchell (1946), Lucas (1977), e Stock e Watson (1993b) para o “estado das artes” em diferentes épocas.

2 Ver Spacov (2000), Issler e Spacov (2000), Chauvet (2001, 2002), Picchetti e Toledo (2002), Duarte, Issler e Spacov (2004), Issler e Notini (2008) e Issler, Notini e Rodrigues (2008). 
de Stock-Watson tradicional. Ao final, a capacidade preditiva dos modelos é comparada no que se refere a datação do ciclo econômico da atividade industrial.

O restante do trabalho se encontra organizado da seguinte forma: na segunda seção, fazemos um breve resumo da literatura; na terceira seção, descrevemos as séries escolhidas; na quarta seção, expomos as técnicas de datação do ciclo econômico; na quinta seção, apresentamos os métodos de construção dos indicadores utilizados; na sexta seção, analisamos os resultados obtidos, e, na sétima seção, concluímos.

\section{RESENHA HISTÓRICA}

Nesta seção realizamos uma pequena resenha histórica sem a pretensão de se exaurir o tema.

O desenvolvimento de indicadores líderes possui origem em 1937, quando o então Secretário do Tesouro, Henry Morgenthau Jr., pediu a Wesley Mitchell que listasse várias séries estatísticas, tentando procurar por indícios de quando a recessão, que se iniciara em 1937, apontava chegar ao fim. Mitchell, em colaboração com Arthur Burns, fez o trabalho e caminhou no sentido de condensar o conhecimento sobre o ciclo de negócios existente na época em um livro de 1946.

Em 1950, Geoffrey Moore, revisando a tal lista, adicionou várias outras séries e, em 1961, Julius Shiskin ${ }^{3}$ desenvolveu a idéia de compor tais séries para a formação de índices antecedentes, coincidentes e atrasados, dando a estrutura que vigora até hoje nesta área de pesquisa.

O National Bureau of Economic Research (NBER), fundado em 1920, passou a datar os ciclos de negócios a partir de 1929; nos dias de hoje, a metodologia do NBER sofisticou-se e alcançou a maturidade com a utilização de métodos de detecção de pontos de inflexão de séries econômicas em geral e outras ferramentas.

Grosso modo, desde 1978, as decisões pautam-se pela análise de várias séries econômicas por um comitê de economistas (Business-Cycle Dating Comittee). Tal análise se dá muito posteriormente ao momento em estudo da economia. Por um lado, assim procedendo, exclui-se a análise equivocada de movimentos espúrios da economia, por outro, é de pouca utilidade para fins de tomada de decisão. Finalmente, em tal reunião, embora técnicas sejam utilizadas no trato das séries, na verdade, não há compromisso com alguma abordagem, a decisão final um acordo entre várias visões pessoais dos especialistas presentes, o que não traz transparência ao processo.

Por seu turno, o The Conference Board (TCB), uma entidade privada e sem fins lucrativos, cuja finalidade é a pesquisa e disseminação de conhecimento na área de Economia, desde 1995, por encomenda do Departamento de Comércio dos Estados Unidos da América, determina

3 Ver Moore e Shiskin (1967). 
uma série de indicadores coincidentes, antecedentes e retardados oficiais. As séries antecedentes, coincidentes e retardadas são, nos EUA, as mesmas daquelas analisadas pelo NBER e em número de 12, 4 e 7, respectivamente. No caso da série coincidente, utilizam-se séries referentes para produção, renda, vendas e emprego. O mesmo padrão é aplicado aos trabalhos do TCB em outros países, cerca de oito países, na elaboração de índices.

O TCB, para a construção de seus índices, utiliza uma média aritmética das séries padronizadas. Neste trabalho, seguimos a metodologia do TCB como uma das abordagens e aplicamos à economia brasileira. Para a formação de índices coincidentes, escolhemos séries semelhantes e, tanto quanto possível, disponíveis por um período longo. Ademais, utilizando as mesmas séries, aplicamos outras metodologias para efeito de comparação.

A abordagem do TCB é, de fato, heurística, embora tenha um grau de acerto apreciável. De outra monta, vários autores propuseram métodos de construção de índices apoiados em técnicas sofisticadas. Posteriormente à técnica do TCB para índices coincidentes e antecedentes, vários autores contribuíram. Stock e Watson (1998a, 1998b, 1998c), por seu turno, propuseram uma modelagem baseada em séries de tempo, tencionando a detecção dos pontos de mudança (turning points) e a construção de índices coincidentes e antecedentes. A modelagem envolvia variáveis não observáveis e observáveis e séries como produção industrial, renda, vendas, emprego. Curiosamente, apesar de sofisticado, o modelo de Stock-Watson falhou em detectar a recessão americana de 1990 e 1991, não obstante adequar-se bem dentro da amostra. Posteriormente o indicador foi aprimorado.

Atualmente, tais técnicas já estão bem disseminadas. Podemos citar, na Europa, o Euro$T C B$, um índice baseado na técnica TCB para países europeus, e o EuroCOIN, de Altissimo et al. (2001), baseado em fator comum, bem como o EuroIJR, que é um fator comum utilizando as séries do TCB.

Avanços recentes incluem a utilização de métodos não lineares. Chauvet (1998), tentando, por um lado, tornar o processo de datação sólido em termos estatísticos e, por outro, aprimorar a detecção de ciclos fora da amostra, vem experimentando a utilização de um modelo de fator comum semelhante com mudança de regime, baseado em cadeias de Markov, tendo tido sucesso em prever a recessão de 1990-1991, da economia americana. O mesmo método de detecção de pontos de mudança de ciclos foi aplicado posteriormente por Chauvet e Piger (2003), ancorados em modelos de Hamilton, na série do próprio PIB americano com resultados interessantes no tocante à previsão.

Um modelo de mudança de regime, baseado em Hamilton (1989) e Lam (1990) foi aplicado no Brasil por Caiado, Céspedes e Chauvet para prever os pontos de mutação de ciclo do PIB industrial, havendo experiência anterior com tais modelos aplicados ao PIB da economia. ${ }^{6}$ No primeiro artigo, são comparadas as habilidades preditivas de modelos lineares e não lineares, com quebras estruturais, para a taxa de crescimento do PIB industrial do Brasil. São estimados

4 Stock e Watson $(1989,1993)$.

5 Ver Brisne et al. (2005).

6 Ver Lima e Domingues (2000). 
os modelos com mudança de regime markoviana propostos por Hamilton (1989) e Lam (1990), que generaliza o modelo do Hamilton. Os resultados sugerem que os modelos não lineares são os que apresentam o melhor desempenho preditivo e que a inclusão de quebras estruturais é importante para se obter a representação do ciclo de negócios no Brasil.

Finalmente, modelos que geram séries de indicadores coincidentes necessitam de que se analise a série em busca dos pontos de mutação. Tais algoritmos determinam o ponto de mutação (o algoritmo de Bry-Boschan destina-se a esse fim, por exemplo), dada uma série qualquer. Formalmente, não são modelos de previsão, mas algoritmo de detecção de pontos de mutação, sendo assim, usualmente prepara-se um indicador, composto por várias séries, e aplica-se o algoritmo. Neste trabalho, utilizaremos o algoritmo de datação de ciclos de Bry-Boschan.

\section{SÉRIES ESCOLHIDAS}

O primeiro passo para a construção dos índices foi a determinação das séries econômicas brasileiras que poderiam ser usadas como variáveis coincidentes para a atividade industrial. A escolha das séries é difícil e depende de algum bom senso e de extensiva análise estatística de correlações e causalidade. Necessitamos de séries longas ao se trabalhar com modelos de fator comum ou modelos de correlações canônicas, mas não com modelos do tipo TCB. Baseados na literatura recente, nós vamos nos apropriar, em boa medida, do trabalho acumulado no Brasil e no exterior.

\subsection{Séries coincidentes}

À luz das séries coincidentes utilizadas pelo TCB, Issler e Spacov (2000) realizaram extensiva pesquisa de séries e concluíram por uma estrutura semelhante à utilizada pelo NBER e pelo TCB, mantendo as equivalências devidas. As séries coincidentes para o PIB, escolhidas por Issler e Spacov (2000), são exibidas no quadro abaixo e tentam capturar a produção, o emprego, as vendas e a renda pessoal:

\section{Quadro 1 - Séries coincidentes para o PIB brasileiro escolhidas por Issler e Spacov (2000)}

\begin{tabular}{|lccl|}
\hline Série & Transformação & Ajuste Sazonal & Fonte \\
\hline Produção Industrial & $\Delta \ln () / \Sigma \ln ()$ & $\operatorname{Sim}$ & PIM/IBGE \\
Emprego & $\Delta \ln () / \Sigma \ln ()$ & $\operatorname{Sim}$ & PME/IBGE e FIESP \\
Expedição de Papelão & $\Delta \ln () / \Sigma \ln ()$ & $\operatorname{Sim}$ & ABPO \\
Renda Pessoal & $\Delta \ln () / \Sigma \ln ()$ & $\operatorname{Sim}$ & PME/IBGE \\
\hline
\end{tabular}

Nota: No trabalho original, cabe comentar, utilizou-se a diferença simétrica para alguns cálculos. 
Na construção de séries coincidentes, utilizamos a mesma idéia inicial, nos permitindo apenas a transposição para o problema do PIB setorial, isto é, sempre que defensável, as séries utilizadas são setorizadas.

Antes vamos tecer algumas considerações. Na série de rendimento efetivo, que representa o poder de compra da população, não caberia a setorização, logo utilizamos a mesma série. Por conseguinte, dada a inexistência de uma série longa, tal como a referência original, reconstruímos a série utilizando a técnica do filtro de Kalman, semelhante do feito em Duarte, Issler e Spacov (2004). Mais precisamente, consideramos um sistema no qual a renda efetiva é função da renda nacional e do PIB a preços de mercado, não sendo observada diretamente. O sistema assim se apresentaria:

$$
\begin{aligned}
& I_{t+1}=\alpha_{1} I_{t}+\alpha_{2} P I B_{t}+\varepsilon_{t+1}, \quad \varepsilon_{t+1} \approx N\left(0, \sigma^{2}\right) \\
& I_{t+1}^{*}=h_{1} I_{t}
\end{aligned}
$$

No caso, $I^{*}{ }_{t}$ e $I_{t}$ são, respectivamente: a renda média real efetivamente recebida pelas pessoas - Região Metropolitana - para o período posterior a setembro de 2001 (período observado) e a renda ao longo de todo o período e, portanto, não observada. O coeficiente $h_{t}$ assume o valor zero para o período não observado e um para o período observado. Para auxiliar na estimação, utilizamos o PIB mensal, série do Banco Central, em valores a preços de mercado, conhecida desde janeiro/1990. Esta técnica se aplica muito bem quando conhecemos parte da série e desconhecemos a série restante.

Com respeito à série representativa do emprego, temos como possibilidade alternativa lançar mão da série de Horas Trabalhadas na Produção, série mantida pela FIESP, que é extensa, desde 1975, mas abrange apenas São Paulo, apesar de ser referente à indústria. De outra monta, a série Horas Trabalhadas na Indústria, da CNI, que é setorial e nacional, contudo, possui apenas dez anos de extensão.

Decidimos por utilizar a série Horas Trabalhadas na Indústria alongada econometricamente, ${ }^{8}$ isso porque as séries são extremamente correlacionadas no período comum.

Finalmente, enquanto a série de expedição de papelão, para a construção de índices coincidentes para o PIB, é uma proxy para as vendas, de fato existe uma série de vendas industriais, que, no entanto, é muito curta, mas apresenta alta correlação com a produção de papel-papelão. Como a série de expedição foi descontinuada e a série de produção de papel e celulose guarda uma alta correlação com esta e abrange um maior período de tempo, resolvemos utilizar esta última como proxy de vendas.

Além disso, utilizamos a série de produção física de Papel e Papelão, descontinuada em 1991, para alongar a série de produção física de Papel, Papelão e Celulose, dado também a alta correlação de ambas.

7 A rotina de Filtro de Kalman se encontra disponível, podendo ser requerida aos autores.

8 Procedimento similar ao realizado com a série de renda. 
Foram escolhidas quatro séries (Produção Industrial; Horas Trabalhadas na Indústria; Produção de Papel, Papelão e Celulose, e Renda) para servirem de variáveis coincidentes da atividade industrial, a partir deste ponto denominadas simplesmente de produção industrial, emprego, vendas e renda. As séries coincidentes escolhidas para o setor industrial são exibidas na Figura 1 abaixo.

Figura 1 - Séries coincidentes (em nível e sem dessazonalizar)

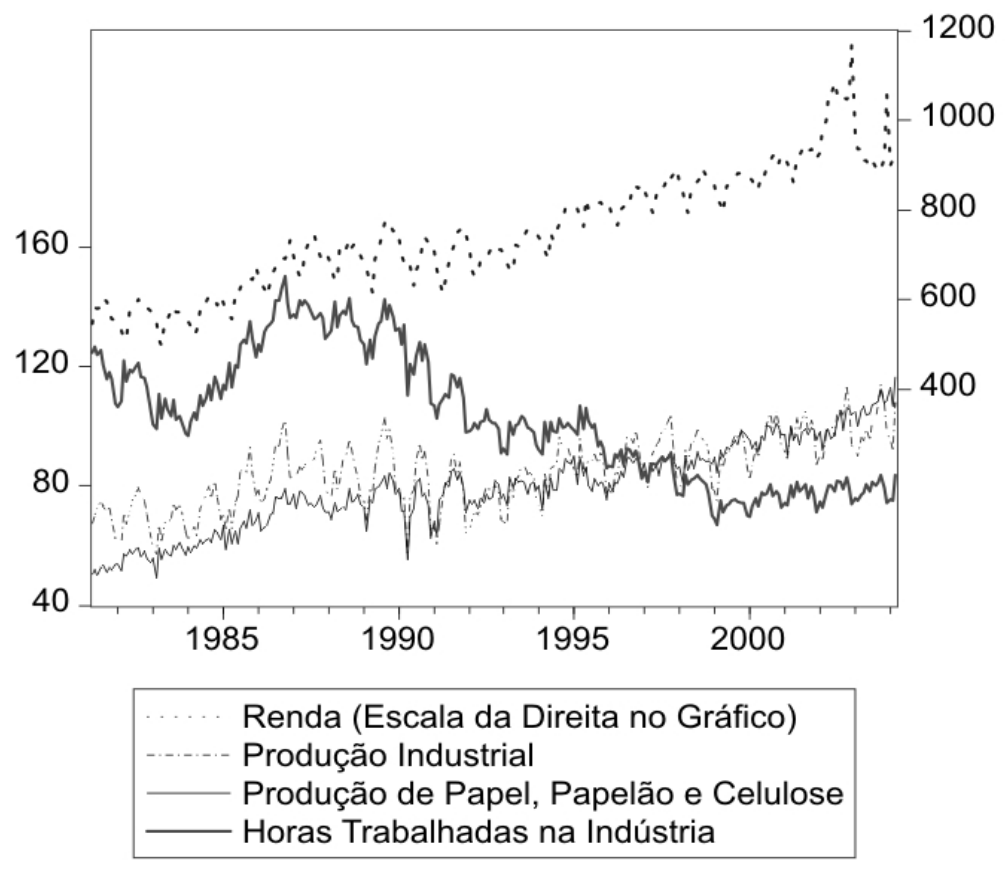

Definidas as quatro séries, em seguida foi tomado o logaritmo das mesmas e imposta uma dessazonalização X-12 aditiva. ${ }^{9}$ Para a construção do indicador baseado na metodologia TCB, foi tomada também a primeira diferença simétrica das séries.

\subsection{Testes de estacionaridade}

Tendo procedido à dessazonalização das séries, passamos a analisar a estacionariedade do conjunto. A estacionaridade das séries coincidentes foi testada utilizando o teste ADF de raiz unitária ajustado pelo critério de informação de Akaike. Os resultados encontram-se na Tabela 1. Como o tamanho amostral das séries é diferente, logo, restringimos o teste ao intervalo comum, a saber, a partir de 1981.6 até 2004.3.

9 Uma descrição detalhada das séries utilizadas e das transformações realizadas se encontra na Tabela A1 do Apêndice. 
Tabela 1 - Teste ADF de raiz unitária

\begin{tabular}{|c|c|c|c|c|c|c|}
\hline & \multicolumn{3}{|c|}{ Em Nível } & \multicolumn{3}{|c|}{ Em diferença } \\
\hline & $t$ & $t_{m}$ & $t_{t}$ & $t$ & $t_{m}$ & $t_{t}$ \\
\hline Produção Ind. & $0.78^{\circ}$ & $-1.34^{\circ}$ & $-3.25^{b}$ & $-9.57^{a}$ & $-9.61^{a}$ & $-9.61^{a}$ \\
\hline Emprego & $-1.14^{c}$ & $-1.10^{\circ}$ & $-1.63^{c}$ & $-6.92^{a}$ & $-7.05^{a}$ & $-7.01^{\mathrm{a}}$ \\
\hline Vendas & $2.14^{\mathrm{c}}$ & $-0.71^{\mathrm{c}}$ & $-3.21^{b}$ & $-8.43^{a}$ & $-8,79^{a}$ & $-8.77^{\mathrm{a}}$ \\
\hline Renda & $1.30^{\circ}$ & $-1.64^{c}$ & $-3.50^{b}$ & $-7.99^{\mathrm{a}}$ & $-8.12^{a}$ & $-8.12^{a}$ \\
\hline
\end{tabular}

Notas: $t$ significa ausência de constante, $t_{m}$ inclusão da constante e $t_{t}$ inclusão de constante e tendência.

a significa que rejeita $H_{0}$ a $1 \%$ de significância.

b significa que rejeita $H_{0}$ a $5 \%$ de significância.

c significa que rejeita $H_{0}$ a $10 \%$ de significância.

Como é possível observar, há evidência de uma raiz unitária nas séries do logaritmo das séries: Produção Industrial, Emprego, Vendas e Renda. Mais ainda, a primeira diferença de cada uma das séries é estacionária, indicando que as séries devem ser integradas de ordem um. Tendo em vista a existência de choques externos, é prudente perfazer o teste de raiz unitária de Perron (1997).

Tabela 2 - Teste de Perron (1997) de raiz unitária

\begin{tabular}{|c|c|c|c|c|c|c|}
\hline & \multicolumn{3}{|c|}{ Em Nível } & \multicolumn{3}{|c|}{ Em primeira diferença } \\
\hline & $t$ & $t_{m}$ & $t_{t}$ & $t$ & $t_{m}$ & $t_{t}$ \\
\hline Produção Ind. & $0.87^{\circ}$ & $-1.77^{c}$ & -4.63 & $-25.13^{a}$ & $-25.11^{a}$ & $-25.08^{a}$ \\
\hline Emprego & $-1.30^{c}$ & $-1.05^{c}$ & $-1.50^{c}$ & $-21.96^{a}$ & $-22.1^{\mathrm{a}}$ & $-22.07^{a}$ \\
\hline Vendas & $1.84^{\mathrm{C}}$ & $-0.98^{c}$ & -4.89 & $-22.35^{\mathrm{a}}$ & $-22.81^{a}$ & $-22.76^{a}$ \\
\hline Renda & $1.30^{\mathrm{C}}$ & $-1.64^{c}$ & $-3.92^{a}$ & $-16.42^{a}$ & $-16.53^{a}$ & $-16.51^{a}$ \\
\hline
\end{tabular}

Notas: $t$ significa ausência de constante, $t_{m}$ inclusão da constante e $t_{t}$ inclusão de constante e tendência.

a significa que rejeita $H_{0}$ a $1 \%$ de significância.

b significa que rejeita $H_{0}$ a $5 \%$ de significância.

c significa que rejeita $H_{0}$ a $10 \%$ de significância.

Com base nos resultados acima, temos que todas as séries são I(1). Logo, o teste ADF e o teste de Perron chegaram às mesmas conclusões. ${ }^{10}$

Finalmente, um último teste que considera o conjunto das séries é o teste de razão da máxima verossimilhança sob a restrição da existência de um vetor de cointegração canônico. A

10 Adicionalmente, foi realizado o teste de raiz unitária proposto por Ng e Perron (2001) e disponível no programa E-views, para cada série coincidente. Os resultados apontam que todas as séries são I(1). Os mesmos podem ser requeridos aos autores. 
imposição de vetores canônicos como vetores de cointegração é efetivamente um teste de raiz unitária. A realização desse teste classificou todas as séries como I(1) ao nível de $1 \%$. $^{11}$

\subsection{Procedimentos de datação}

Parte do trabalho de construção de indicadores coincidentes constitui-se em definir o que se entende por recessão ou ciclo de crescimento e se o processo pode prever fora da amostra com relativo sucesso, tais são os dois problemas da datação.

As definições ciclo de crescimento ou de recessão ao olhar da NBER são: por recessão, entende-se um declínio coordenado de várias grandezas, não somente o PIB, por, geralmente, seis meses. ${ }^{12}$ Tal coordenação se verifica tanto em recessão quanto em crescimento, segundo as caracterizações dos ciclos de negócios conhecidas. ${ }^{13}$ A questão, então, que se deriva naturalmente, seria identificar os fatores líderes de processo, evitando fatores secundários, e monitorando um conjunto pequeno de fatores e, principalmente, compondo-os corretamente. Neste sentido, o trabalho seminal de Mitchell e Burns permanece atual.

A interpretação dos estados da economia é naturalmente matéria controversa. Dada a inexistência de um órgão no Brasil que tome para si a tarefa de classificação da economia em estados de recessão ou expansão, a leitura do indicador coincidente é dificultada na medida em que é subjetiva.

Uma maneira de se dirimir tal subjetividade é lançar mão de algoritmos de datação, notando que duas funções tais algoritmos devem ter. A primeira consiste simplesmente em classificar corretamente os estados econômicos ao longo da série. A segunda, se possível, oferecer a possibilidade de detecção dos futuros estados da economia. Neste trabalho, utilizaremos o Algoritmo de Bry-Boschan para a datação dos ciclos de crescimento e recessão. Uma descrição detalhada do procedimento de datação proposto por Bry e Boschan se encontra no Apêndice.

\section{METODOLOGIAS UTILIZADAS DE CONSTRUÇÃO DOS INDICADORES COINCI- DENTES}

Nesta seção, serão apresentados os quatro procedimentos utilizados na construção dos indicadores coincidentes.

\subsection{Metodologia TCB}

Como já pontuado, as séries antecedentes, coincidentes e de retardados são, nos EUA, as mesmas daquelas analisadas pelo NBER e em número de 12,4 e 7 , respectivamente. No caso

11 Os resultados do teste podem ser requeridos aos autores.

12 Ver http://www.nber.org/cycles.html

13 Ver Lucas (1977). 
da série coincidente, utilizam-se séries referentes para produção, renda, vendas e emprego. $\mathrm{O}$ mesmo padrão é aplicado aos trabalhos do TCB em outros países, cerca de oito países, na elaboração de índices. O TCB, para a construção de seus índices, utiliza uma média aritmética das séries padronizadas. A padronização segue quatro passos.

Calculam-se as diferenças mensais das séries que compõem o índice. Se a série $\left\{X_{t}\right\}$ está em porcentagem ou é uma taxa de juros, simplesmente faz-se a diferença. Caso contrário, utiliza-se a fórmula de diferença simétrica $x_{t}=200 \cdot \frac{X_{t}-X_{t-1}}{X_{t}+X_{t-1}}$

As diferenças mensais são ajustadas pela volatilidade. Os desvios padrão $v \mathrm{x}$ das diferenças mensais das séries são calculados. Seja k o somatório dos inversos dos desvios padrão, $k=\sum_{x} 1 / v_{x}$, a série de diferenças ajustada é dada por $m_{t}=\frac{x_{t}}{k v_{x}} ;$

Computa-se a soma das diferenças mensais ajustadas: $i_{t}=\sum_{x} m_{t}$

$\mathrm{O}$ índice é calculado usando-se a fórmula das diferenças simétricas. O valor do índice correspondente ao primeiro mês é $I_{t}=\frac{200+i_{1}}{200-i_{1}}$, a partir do segundo mês, temos $I_{t}=\frac{200+i_{t}}{200-i_{t}} I_{t-1}$.

A cada novo dado, os desvios padrão são atualizados e a série inteira é recalculada.

\subsection{Construção de indicadores TCB com modelagem das heterocedasticidades}

Uma pergunta natural seria se, ao invés de se utilizar uma volatilidade padronizadora constante para toda a amostra, utilizássemos uma variável com o tempo. Lumsdaine e Prasad (2003) sugeriram modelar o peso em uma abordagem tipo TCB, mas pertinente a outro problema, utilizando um GARCH $(1,1)$. Neste trabalho, tentaremos algo semelhante, porém, de modo a levar em conta o comportamento cíclico das variáveis, utilizaremos um modelo de ordem superior, ajustando na amostra por meio de critérios de informação. O ponto fundamental é modelar a influência dos pesos de modo contemporâneo. O modelo é apresentado abaixo:

$$
\begin{aligned}
& \varphi(L) y_{t}=\varepsilon_{t}, \quad \varepsilon_{t} \mid I_{t-1} \approx N\left(0, \sigma_{t}^{2}\right) \\
& \sigma_{t}^{2}=w+\alpha \varepsilon_{t-1}^{2}+\beta \sigma_{t-1}^{2}
\end{aligned}
$$

\subsection{Metodologia Stock-Watson}

A metodologia Stock-Watson fundamentalmente é um modelo paramétrico que tenta capturar o movimento simultâneo das variáveis selecionadas e obter a partir deste um indicador coincidente. Essas variáveis são, então, pensadas como decomponíveis em um fator comum não observável e um fator idiossincrático. O modelo pode, então, ser colocado em um modelo espaço 
de estado e, como usual, o estado não observável pode ser filtrado e suavizado com rotinas de Kalman, sendo este o indicador coincidente.

Em seguida, apresentamos o modelo de Stock-Watson padrão:

$$
\begin{aligned}
& \Delta X_{t}=\beta+\gamma(L) \Delta C_{t}+u_{t} \\
& D(L) u_{t}=\varepsilon_{t} \\
& \Delta C_{t}=\mu_{c}+\lambda_{c c} \Delta C_{t-1}+\lambda_{c y} Y_{t-1}+\nu_{c t} \\
& Y_{t}=\mu_{Y}+\lambda_{y c} \Delta C_{t-1}+\lambda_{y y} Y_{t-1}+\nu_{y t}
\end{aligned}
$$

As séries coincidentes são coletadas no vetor $X_{t}$, sendo o índice coincidente $C_{t}$ e $Y_{t}$ o indicador antecedente. As equações (7) e (8) estabelecem a ligação entre o índice coincidente, $C_{t}$, e o antecedente $Y_{t}$. No trabalho original, não havia essa preocupação e, no nosso caso, pode ser ignorado o componente antecedente. Os erros são supostos não correlacionados e a estimação dos parâmetros é realizada por critérios estatísticos.

O ponto fundamental é que, nesta formulação, há uma variável não observada que é conhecida utilizando-se o aparato de Kalman. ${ }^{14}$ Posteriormente, pode-se realizar uma previsão para tais índices coincidentes utilizando-se as duas últimas equações. Neste trabalho, realizamos a construção de um indicador Stock-Watson para o PIB industrial.

\subsection{Modelo de Vetores Autorregressivo de Frequência Mista}

Recentemente, Mariano e Murasawa (2003) observaram que, apesar de ser o indicador agregado mais importante em qualquer diagnóstico da economia, o PIB trimestral não contribuía, direta ou indiretamente, para a confecção de indicadores coincidentes.

A bem da verdade, esta omissão pode advir do fato de que, mesmo trimestralmente conhecido, nem sempre é de pronto conhecimento. Ocorre que, na classificação dos estados passados da economia e na aferição de outros índices coincidentes, poderia ser de extrema utilidade, mais ainda quando optamos por realizar índices coincidentes setoriais da economia.

O problema posto, porém, é que as séries coincidentes são mensais e as séries existentes, no caso do PIB industrial, são trimestrais, e alguma composição é necessária. A idéia de se combinar dados medidos em diferentes frequências, em um modelo de regressão, é relativamente nova. A literatura chama este tipo de regressão de Mixed Data Sampling Regression (MIDAS), sendo que existem várias formas de se construir tais regressões.

14 A rotina de filtro de Kalman utilizada para a obtenção do indicador coincidente de Stock-Watson foi desenvolvida por Mariano-Murasawa e encontra-se disponível no site do Journal of Applied Econometrics. 
Regressões MIDAS envolvem regressores com diferentes frequências amostrais, logo não são modelos autorregressivos, visto que autorregressão assume que os dados são medidos na mesma frequência no passado. Na verdade, regressões MIDAS possuem algumas características típicas de modelos de atraso distribuído (distributed lag models). ${ }^{15}$

Mariano e Murasawa (2003) propuseram uma análise de fator por máxima verossimilhança para circunscrever o problema que possui convergência difícil. O modelo de MarianoMurasawa, por não se utilizar de uma regressão, a princípio, não pertence à classe de modelos MIDAS. A seguir, iremos descrever o modelo de Mariano-Murasawa, no qual foi proposta uma estrutura VAR sem qualquer imposição de fator único.

\subsubsection{Modelo VAR}

Seja $\left\{\mathrm{Y}_{1, \mathrm{t}}\right\}_{\mathrm{t}=-\infty, \infty}$ uma série $\mathrm{Nx}$ 1 aleatória trimestral qualquer e $\left\{\mathrm{Y}_{2, \mathrm{t}}\right\}_{\mathrm{t}=-\infty, \infty}$ uma série $\mathrm{Mx}$ 1, mensal, aleatória. Seja $\mathrm{NM}=\mathrm{N}+\mathrm{M}$. Ademais, seja $\left\{\mathrm{Y}_{1, \mathrm{t}}^{*}\right\}_{\mathrm{t}=-\infty, \infty}$ a série aleatória latente correspondente. Tal que:

$$
\ln Y_{1, t}=\frac{1}{3}\left(\ln Y_{1, t}^{*}+\ln Y_{1, t-1}^{*}+\ln Y_{1, t-2}^{*}\right)
$$

Neste caso, a variável observável, a cada terceiro mês, é a média geométrica das variáveis latentes. Tomando a diferença em três períodos, chegamos a:

$$
\ln y_{1, t}=\frac{1}{3} \ln y_{1, t}^{*}+\frac{2}{3} \ln y_{1, t-1}^{*}+\ln y_{1, t-2}^{*}+\frac{2}{3} \ln y_{1, t-3}^{*}+\frac{1}{3} \ln y_{1, t-4}^{*}
$$

onde $y_{1, t} \equiv \Delta_{3} \ln Y_{1, t}$ e $y_{1, t}^{*} \equiv \Delta \ln Y_{1, t}^{*}$

Observamos $y_{1, t}$ todo o terceiro mês e nunca observamos $y_{1, t}^{*}$. Para todo t, seja:

$$
y_{t} \equiv\left(\begin{array}{l}
y_{1, t} \\
y_{2, t}
\end{array}\right), y_{t}^{*} \equiv\left(\begin{array}{l}
y_{1, t}^{*} \\
y_{2, t}
\end{array}\right)
$$

onde $y_{2, t}=\Delta \ln Y_{2, t}$. Assumindo um modelo de um único fator estático para $\left\{y_{t}^{*}\right\}_{-\infty}^{\infty}$, tal que, para todo t, temos:

$$
\begin{aligned}
& \left(\begin{array}{l}
y_{1, t}^{*} \\
y_{2, t}
\end{array}\right)=\left(\begin{array}{l}
\mu_{1}^{*} \\
\mu_{2}
\end{array}\right)+\beta f_{t}+u_{t} \\
& \varphi_{f}(L) f_{t}=v_{1, t} \\
& \Phi_{u}(L) u_{t}=v_{2, t}
\end{aligned}
$$

15 Para maiores detalhes sobre os modelos MIDAS, ver Ghysels et al. (2003). 


$$
\left(\begin{array}{l}
v_{1, t} \\
v_{2, t}
\end{array}\right) \sim N I D\left(\begin{array}{cc}
\sigma_{1}^{2} & 0 \\
0, & \\
0 & \sum 22
\end{array}\right)
$$

onde $\beta \in \mathfrak{R}^{N}$ é o vetor de fatores, $\left\{f_{t}\right\}_{-\infty}^{\infty}$ é uma sequência estacionária de fatores comuns, $\left\{u_{t}\right\}_{-\infty}^{\infty}$ é uma sequência estacionária de fatores específicos, L é o operador de atraso, $\varphi_{f}($.$) é um polinômio de ordem p e \Phi_{u}($.$) é um polinômio de ordem q. Para identificar o$ modelo, Mariano e Murasawa (2003) assumiram que o primeiro componente de $\beta$ é um e que $\Phi_{u}($.$) e \Sigma 22$ são diagonais. Considere o seguinte operador de atraso:

$$
\begin{aligned}
& H(L)=\left[\begin{array}{cc}
(1 / 3) I_{N} & 0 \\
0 & I_{M}
\end{array}\right]+\left[\begin{array}{cc}
(2 / 3) I_{N} & 0 \\
0 & 0
\end{array}\right] L+\left[\begin{array}{cc}
I_{N} & 0 \\
0 & 0
\end{array}\right] L^{2}+\left[\begin{array}{cc}
(2 / 3) I_{N} & 0 \\
0 & 0
\end{array}\right] L^{3}+ \\
& {\left[\begin{array}{cc}
(1 / 3) I_{N} & 0 \\
0 & 0
\end{array}\right] L^{4}}
\end{aligned}
$$

Podemos então reescrever a equação (12) da seguinte forma:

$$
(y-\mu)=H(L)\left(y_{t}^{*}-\mu^{*}\right)
$$

\subsubsection{Representação em espaço de estado}

Vamos assumir a representação de estado-espaço de maneira a calcular os estados não observáveis via estimação de Kalman para p $<5$. Mariano e Murasawa (2003) completaram as observações mensais que estavam faltando à série trimestral com zeros, de modo a poder aplicar o filtro de Kalman na avaliação da função de verossimilhança. A seguir, iremos fazer uma breve descrição do modelo de estado-espaço desenvolvido por Mariano e Murasawa (2003). Seja $S_{t}$ o vetor de estados no instante $t$ :

$$
S_{t}=\left(\begin{array}{c}
f_{t} \\
\vdots \\
f_{t-5} \\
u_{t} \\
\vdots \\
u_{t-5}
\end{array}\right)
$$


O modelo estado-espaço é, então, descrito pelas equações (19) e (20):

$$
\begin{aligned}
& S_{t+1}=A S_{t}+B z_{t} \\
& y_{t}=\mu+C S_{t}
\end{aligned}
$$

As equações (19) e (20) representam, respectivamente, a equação de estado e a equação de observação do modelo. O erro da equação de estado $z_{t}$ se distribui como uma normal independente e identicamente distribuída $\left(z_{t} \approx N I D\right)$. Sendo que as matrizes A, B e C são, respectivamente:

$$
\begin{aligned}
& A=\left[\begin{array}{ccc}
\Phi_{1} & \ldots . \Phi_{p} & O_{N x(5-P) N} \\
& O_{4 N x N} \\
I_{4 N x N} & O_{4 N x N}
\end{array}\right] \\
& B=\left[\begin{array}{c}
\Sigma^{1 / 2} \\
O_{4 N x N}
\end{array}\right] \\
& C=\left[H_{0} \ldots . . H_{4}\right]
\end{aligned}
$$

A estimação é realizada via método de Quase-Newton e o Algoritmo de maximização de esperança é descrito em Mariano e Murosawa (2003).

\section{RESULTADOS DENTRO DA AMOSTRA}

Para efeitos de comparação, nós vamos nos limitar ao intervalo comum da amostra, 1981.62004.3, contrapondo as diversas metodologias.

Na Figura 2, são apresentados os indicadores coincidentes, construídos com base na metodologia TCB, TCB modificado e Stock-Watson. O indicador TCB modificado foi construído modelando-se um GARCH(2,2). ${ }^{16} \mathrm{Na}$ Figura 3, é apresentado o indicador coincidente de Mariano-Murasawa.

16 A escolha da ordem do modelo se deu através da utilização do critério de informação de Schwartz. 
Figura 2 - Indicadores coincidentes TCB, TCB modificado e Stock-Watson

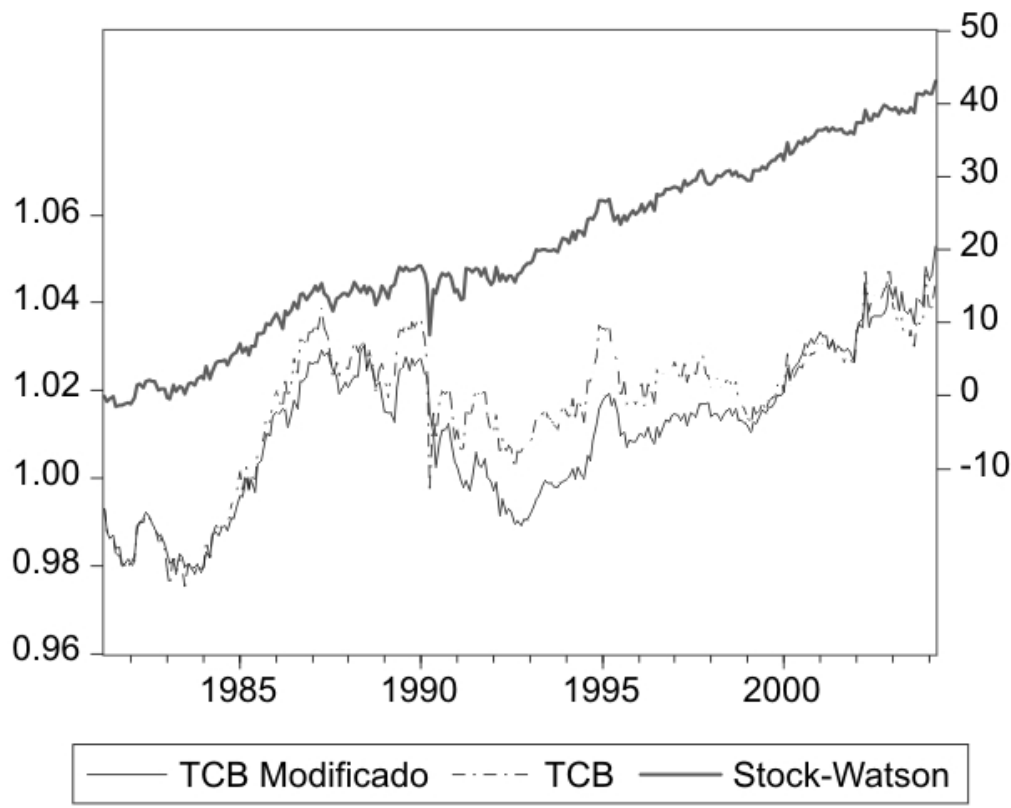

Figura 3 - Indicador coincidente de Mariano-Murasawa

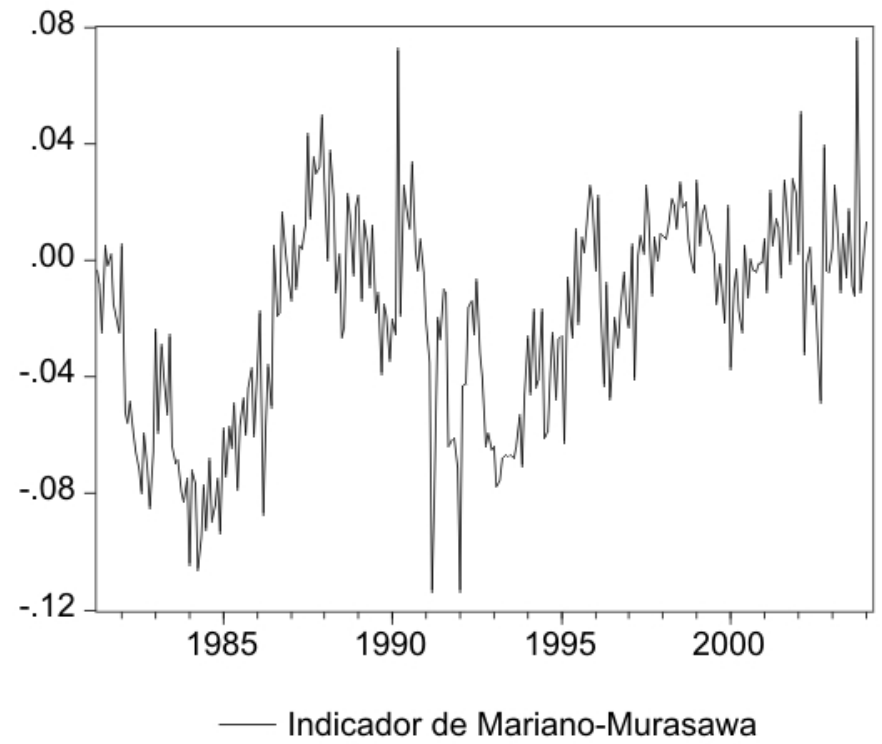

Como podem ser observados pela Figura 2, os indicadores TCB e TCB modificado têm comportamento bastante similar. Logo, é de se esperar que a datação aplicada a cada um dos 
índices terá resultados semelhantes. Já os estimadores de Stock-Watson e Mariano-Murasawa possuem comportamentos muito distintos entre si e se comparados com os indicadores baseados na metodologia TCB.

Uma vez construídos os quatro indicadores coincidentes, aplicamos a rotina de Bry-Boschan a cada um deles, de modo a datar os ciclos econômicos da produção industrial brasileira. Como no Brasil não existe um órgão responsável pela datação do ciclo econômico, como nos EUA, recorremos a uma análise visual de modo a comparar a performance de cada um dos indicadores propostos em descrever o estado (recessão/expansão) da produção industrial brasileira. Isso foi feito da seguinte forma: aplicamos a datação sugerida por cada índice ao conjunto de séries que o compõem (o que inclui a própria produção industrial). A datação que descrever melhor os ciclos das séries e principalmente a série de produção industrial será a escolhida. O exercício descrito se encontra nas Figuras A3, A4 e A5 do Apêndice.

Embora muito similar ao TCB modificado, a datação do índice TCB apresentou maior número de pontos de reversão. Já o indicador Stock-Watson foi incapaz de captar vários períodos recessivos. Finalmente, o indicador de Mariano-Murasawa apresentou datação semelhante ao método TCB, mas se mostrou mais lento em captar os ciclos econômicos. Deste modo, a escolha recai sobre o indicador TCB por ser eficiente na datação do ciclo industrial e pela simplicidade de sua implementação. A datação sugerida pelo TCB se encontra na Tabela 3.

\section{Tabela 3 - Datação Bry-Boschan do Índice Coincidente TCB}

\begin{tabular}{cc}
\hline Picos & Vales \\
\hline 1982 M06 & 1982 M01 \\
1987M04 & 1983 M07 \\
1990 M01 & 1989 M02 \\
1991 M07 & 1991 M02 \\
1995 M03 & 1992 M08 \\
1997 M10 & 1996 M03 \\
2001 M03 & 1999 M02 \\
2002 M12 & 2001 M12 \\
& 2003 M08 \\
\hline
\end{tabular}

A partir da Tabela 3, podemos concluir que a atividade industrial sofreu nove recessões no período de junho de 1981 a março de 2004. Em média, observamos uma recessão a cada dois anos e quatro meses, o que pode ser considerado alto vis-à-vis aos EUA, que passaram em média por uma recessão a cada cinco anos. As recessões brasileiras também duraram mais tempo se comparadas com as americanas: no Brasil, uma recessão dura em média 12 meses, enquanto que nos EUA dura seis meses. 
Em seguida, comparamos os quatro índices coincidentes construídos com base na correlação cruzada existente entre eles.

\section{Tabela 4 - Tabela de correlação cruzada}

\begin{tabular}{lcccc}
\hline & Mariano-Murasawa & Stock-Watson & TCB Padrão & TCB Modificado \\
\hline Mariano-Murasawa & 1 & 0.489194 & 0.553992 & 0.613305 \\
Stock-Watson & 0.489194 & 1 & 0.782102 & 0.776957 \\
TCB Padrão & 0.553992 & 0.782102 & 1 & 0.924795 \\
TCB Modificado & 0.613305 & 0.776957 & 0.924795 & 1 \\
\hline
\end{tabular}

A partir da Tabela 4, podemos concluir que o indicar TCB e o indicador TCB modificado apresentam uma alta correlação, apresentando informações semelhantes sobre o estado da economia. O indicador coincidente de Mariano-Murasawa apresenta a menor correlação com os demais indicadores, logo, a princípio, traz informações bastante distintas dos demais.

\section{CONCLUSÃo}

Este artigo propõe e compara quatro índices coincidentes para a atividade industrial brasileira. Especificamente, testou-se uma versão baseada no indicador coincidente do The Conference Board (TCB), uma versão modificada deste, na qual as volatilidades são modeladas; a abordagem de Stock-Watson tradicional e, finalmente, testou-se a abordagem de MarianoMurasawa, que permite a construção de um índice coincidente a partir de séries de dupla frequência.

O índice de Mariano e Murosawa (2003) dá alento à ideia de se agregar informação trimestral à pesquisa de Indicadores Coincidentes, visto ser competitivo com o TCB padrão, sendo, contudo, muito mais sofisticado. O TCB, no entanto, é mais ágil na identificação de períodos recessivos, enquanto que o índice de Mariano-Murasawa tende a agregá-los e a ser mais lento em sua identificação dentro da amostra, deixando alguns pequenos períodos recessivos de lado.

A utilização de um modelo de frequência mista dá, porém, um refúgio ao método TCB, na medida em que confirma a construção de índices setoriais. Isto é, dado que o índice TCB é essencialmente heurístico, a sua aproximada confirmação por um método que utilize informação setorial trimestral é uma contribuição importante e também uma autorização para o método heurístico. O método de Stock e Watson não é adequado, pois não indica quase nenhum período.

De acordo com a datação do índice coincidente TCB, a produção industrial brasileira passou por nove recessões no período entre 1981:6 e 2004:3. Em média, observamos uma recessão a cada dois anos e quatro meses, o que pode ser considerado alto vis-à-vis aos EUA, que passa- 
ram, em média, por uma recessão a cada cinco anos. As recessões brasileiras também duraram mais tempo se comparadas com as americanas: no Brasil, uma recessão dura em média 12 meses enquanto que nos EUA dura seis meses. Há que se levar em conta, entretanto, o excepcional período em que foram aplicadas essas técnicas, que inclui a chamada "década perdida".

Finalmente, uma possível extensão natural do trabalho é criar um indicador antecedente que agregue informações trimestrais.

\section{REFERÊNCIAS}

ALTISSIMO, F. et al. Eurocoin: a real time coincident indicator of the euro area business cycle. CEPR, 2003. (Discussion Paper, n. 242).

AUERBACH, A. J. The index of leading indicators: measurement without theory thirty-five years later. Review of Economics and Statistics, v. 64, n. 4, p. 589-595, 1982.

BERNARD, H.; GERLACH, S. Does the term structure predict recessions? The international evidence. International Journal of Finance and Economics, n. 3, p. 195-215, 1998.

BIRCHENHALL, C. R.; OSBORN, D. R.; SENSIER, M. Predicting UK business cycle regimes. Scottish Journal of Political Economy, v. 48, n. 2, p. 179-95, 2001.

BOIVIN, J.; NG, S. Are more data always better for factor analysis? Cambridge: NBER, 2003. (Working Paper, n. 9.829).

BOLDIN, M. D. Dating turning points in the business cycles. Journal of Business, New York, v. 67, n. 1, p. 97-130, 1994.

BRISNE, J. et al. Forecasting Brazilian output in real time in the presence of breaks: a comparison of linear and nonlinear models. Estudos Econômicos, v. 36 , n. 1, p. 5-46, 2006.

BRY, G.; BOSCHAN, C. Cyclical analysis of time séries: selected procedure and computer programs. New York: National Bureau of Economics Research (NBER), 1971.

BURNS; Mitchell. Measuring business cycles. New York: NBER, 1946.

CAMBA-MENDEZ, G. et al. An automatic leading indicator of economic activity: forecasting GDP growth for European countries. Econometrics Journal, n. 4, p. S56-S90, 2001.

CHAUVET, M. An econometric characterization of business cycle dynamics with factor structure and regime switching. International Economic Review, n. 39, p. 969-996, 1998.

CHAUVET, M.; PIGER, J. M. Identifying business cycle turning points in real time. Review Federal Reserve Bank of St. Louis, n. 0, p. 47-61, 2003.

CHAUVET, M.; SILVA, J. A. B. da. Indicadores antecedentes de recessões brasileiras. In: XXVI ENCONTRO BRASILEIRO DE ECONOMETRIA. João Pessoa, 10, 11, 12 dez. 2004.

CHAUVET, M.; LIMA, E.; VASQUEZ, B. Forecasting Brazilian output in real time in the presence of breaks: a comparison of linear and non-linear models. Rio de Janeiro: Ipea, 2002. (Texto para Discussão, n. 11).

CONTADOR, R. C. Ciclos econômicos e indicadores de atividade. Rio de Janeiro: INPES/IPEA, 1977. 237 p.

CONTADOR, C.; FERRAZ, C. Previsão com indicadores antecedentes. Rio de Janeiro: Silcon, 1999. 
CORREA, A. S. Diferenças entre países da América Latina: uma análise de Markov-Switching para os ciclos econômicos de Brasil e Argentina. Brasília: Banco Central do Brasil, 2003. (Trabalho para Discussão, n. 80).

DUARTE, A. J.; ISSLER, J. V.; SPACOV, A. D. Indicadores coincidentes de atividade econômica e uma cronologia de recessões para o Brasil. Pesquisa e Planejamento Econômico, v. 34, n. 1, p. 1-37, 2004.

DUEKER, M. J. Dynamic forecasts of qualitative variables: a Qual VAR model of US recessions. Journal of Business and Economic Statistics, v. 23, n. 1, p. 96-104, Jan. 2005.

DUEKER, M. J.; WESCHE, K. Forecasting output with information from business cycle turning points: a qualitative variable VAR. Federal Reserve Bank of St. Louis, 2001. (Working Paper).

ESTRELLA, A.; MISHKIN, F. S. Prediciting U.S. recessions: financial variables as lead-ing indicators. Review of Economics and Statistics, n. 80, p. 45-61, 1999.

. The predictive power of the term structure of interest rates in Europe and the United States: implications for the European Central Bank. European Economic Review, n. 41, p. 1.375-1.401, 1997.

ESTRELLA, A.; RODRIGUES, A. P.; SCHICH, S. How stable is the predictive power of the yield curve? Evidence from Germany and the United States., 2000. (Federal Reserve Bank of New York Staff Report, n. 113).

FORNI, M.; LIPPI, M. The generalized factor model: representation theory. Econometric Theory, n. 17, p. 1.113-1.141, 2001.

FORNI, M. et al. The generalized dynamic factor model, one-sided estimation and fore-casting. 2003. Disponível em: <http://homepages.ulb.ac.be/ lreichli/papers/fhlrforec.pdf>. Acesso em: 2004.

GHYSELS, E.; SANTA-CLARA, P.; SINKO, A.; VALKANOV, R. MIDAS regressions: further results and new directions. UNC; UCLA, 2003. (Working Paper).

HAMILTON, J. D. A new approach to the economic analysis of nonstationary time séries and the business cycle. Econometrica, v. 57, n. 2, p. 357-84, Mar. 1989.

INKLAAR, R.; JACOBS, J.; ROMP, W. Business cycle indexes: does a heap of data help? Nederland: University of Groningen, Sept. 2004.

ISSLER, J. V.; NOTINI, H. H. Estimating Brazilian monthly real PIB: a Kalman filter approach. In: CIRET, 2008. Graduate School of Economics; Getulio Vargas Foundation, 2008. Mimeo.

ISSLER, J. V.; NOTINI, H. H.; RODRIGUES, C. F. Evaluating Different Approachs in Constructing Coincident and Leading Indices in Economic Activity for the Brazilian Economy. In: CIRET, 2008. Graduate School of Economics; Getulio Vargas Foundation. Mimeo.

ISSLER, J.; SPACOV, A. Usando correlações canônicas para identificar indicadores antecedentes e coincidentes da atividade econômica no Brasil. 2000. (Relatório de Pesquisa para o Ministério da Fazenda).

LAM, P. S. The Hamilton model with a general autoregressive component. Journal of Monetary Economics, v. 26, p. 409-432, 1990.

LIMA, E. C. R.; DOMINGUES, G. B. Crescimento, recessão e probabilidade de reversão do ritmo de crescimento econômico do Brasil. Boletim Conjuntural, Rio de Janeiro, v. 51, p. 49-52, 2000.

LUCAS, R. E. Understanding business cycle. Carnegie-Rochester Conference Séries on Public Policy, n. 5, p. 7-29, 1977.

LUMSDAINE, R. L.; PRASAD, E. S. Identifying the common component of international economic fluctuations: a new approach. The Economic Journal, Blackwell Synergy, n. 113, p. 101-127, 2003.

LUTKEPOHL, H. Forecasting aggregated vector arma processes. Berlin: Springer-Verlag, 1987. 
MARCELLINO, M. Leading indicators: what have we learned? Igier: Universitá Bocconi, March, 2005. (Working Paper, n. 286). Disponível em: <http://www.igier. uni-bocconi.it>. Acesso em: 2004.

MARCELLINO, M.; STOCK, J. H.; WATSON, M. W. A comparison of direct and indirect multistep ar methods for macroeconomics séries. Innocenzo Gasparini Institute for Economic Research (Igier), Bocconi University, Fev. 2004. (Working Paper, n. 285).

MARIANO, R.; MURASAWA, Y. A new coincident index of business cycles based on monthly and quarterly series. Journal of Applied Econometrics, n. 18, 427-43, 2003.

MITCHELL, W. C.; BURNS, A. F. Statistical indicators of cyclical revivals. NBER Bulletin, New York, n. 69, 1938. Reprinted as Chapter 6 of MOORE, G. H. (Ed.). Business Cycle Indicators. Princeton: Princeton University Press, 1961.

MONETA, F. Does the yield spread predict recessions in the Euro area? International Finance, v. 8, n. 2, p. 263-301, Summer 2005.

MOORE, G. H.; SHISKIN, J. Indicators of business expansions and contractions. National Bureau of Economic Research, Incorporated, Jan. 1967.

NEFÇI, S. Optimal prediction of cyclical downturn. Journal of Economic Dynamics and Control, Aug. 1982.

NG, S.; PERRON, P. LAG length selection and the construction of unit root tests with good size and power. Econometrica, v. 69, n. 6, p. 1519-1554, Nov. 2001.

PERRON, P. The great crash, the oil price shock, and the unit root hypothesis. Econometrica, v. 57, n. 6, p. 1361, 1997.

OSBORN, D.; SENSIER, M.; SIMPSON, P. W. Forecasting UK industrial production over the business cycle. Journal of Forecasting, v. 20, n. 6, p. 405-24, 2001.

PICCHETTI, P.; TOLEDO, C. Estimating and interpreting a common stochastic component for the Brazilian industrial production index. Revista Brasileira de Economia, v. 56, p. 107-20, 2002.

SARGENT, T. J.; SIMS, C. A. Business cycle modeling without pretending to have too much a priori economic theory. In: SIMS, C. A. (Ed.). New methods in business research. Minneapolis: Federal Reserve Bank of Minneapolis, 1977.

SPACOV, A. D. Índices antecedentes e coincidentes da atividade econômica brasileira: uma aplicação da análise de correlação canônica. 2001. Dissertação (Mestrado em Economia) - Orientado por João Victor Issler.

STOCK, J. H.; WATSON, M. W. New indexes coincident and leading economic indicators, NBER: Macroeconomics Annual. Cambridge: MIT Press, 1989.

. A probability model of the coincidence indicators. In: LAHIRI, K.; MOORE, G. H. (Ed.). Leading economic indicators: new approaches and forecasting records. Cambridge, UK: Cambridge University Press, 1991.

. A procedure for predicting recessions with leading indicators: econometric issues and recent experience. Cambridge: NBER, 1992. (Working Paper Séries, n. 4.014).

. A procedure for predicting recessions with leading indicators: econometric issues and recent experience. In: STOCK, J., WATSON, M. (Ed.). New research on business cycles, indicators and forecasting. Chicago: University of Chicago Press, 1993.

. A new indexes of coincident and leading economics indicators. Macroeconmics Annual, NBER, p. $351-95,1998$ a. 
. A new approach to leading economic indicators. Harvard University, Kenedy School of Government, 1998b. Mimeo.

. A probability model of the coincident economic indicators. Cambridge: NBER, 1998c. (Working Paper, n. 2.772).

\section{APÊNDICE A1}

Tabela A1 - Séries utilizadas neste trabalho para a confecção de um índice coincidente

\begin{tabular}{llll}
\hline & Transformação & Ajuste Sazonal & Fonte \\
\hline Produção Industrial & $\Delta \ln () / \Sigma \ln ()^{*}$ & Sim & PIM/IBGE \\
Horas Trabalhadas na Indústria ** & $\Delta \ln () / \Sigma \ln ()^{*}$ & $\operatorname{Sim}$ & $\mathrm{CNI}$ \\
Produção Física de Papel, Papel e Celulose $^{* *}$ & $\Delta \ln () / \Sigma \ln ()^{*}$ & $\operatorname{Sim}$ & IBGE \\
Rendimento Efetivo Real** $^{*}$ & $\Delta \ln () / \Sigma \ln ()^{*}$ & $\operatorname{Sim}$ & PME/IBGE \\
\hline
\end{tabular}

Notas: o ‘**' assinala séries que foram remontadas e ‘*' assinala que o TCB e o TCB modificado utilizaram a diferença simétrica, enquanto que o Stock-Watson e Mariano-Murasawa não.

\section{APÊNDICE A2 - Algoritmo DE BRY-Boschan}

Este algoritmo foi concebido para detectar os pontos de inflexão de séries de tempo. Sumariamente, os passos são os seguintes:

1. Determinam-se os outliers (tendo como base 3,5 desvios padrão da média), substituindo-os por valores calculados pelos derivados por uma curva de Spencer, basicamente uma média móvel das sete observações passadas e futuras. Explicitamente, a expressão completa é:

$$
\begin{aligned}
& S_{t}=\frac{1}{320}\left(-3 x_{t-7}-6 x_{t-6}-5 x_{t-5}+3 x_{t-4}+21 x_{t-3}+46 x_{t-2}+\right. \\
& \left.67 x_{t-1}+74 x_{t}+67 x_{t+1}+46 x_{t+2}+21 x_{t+3}+3 x_{t+4}-5 x_{t+5}-6 x_{t+6}-3 x_{t+7}\right)
\end{aligned}
$$

2. Determinam-se os máximos e mínimos locais na série de média-móvel de 12 meses:

a. Considera-se máximo (mínimo) local a observação que é maior (menor) que as cinco anteriores e posteriores.

b. Caso haja dois máximos (mínimos) consecutivos, seleciona-se o maior (menor).

3. Os pontos de inflexão determinados no passo anterior são refinados com a curva de Spencer:

a. Para cada máximo (mínimo) determinado no passo anterior, seleciona-se a maior (menor) observação na curva de Spencer, que dele diste até 5 observações (ou seja, meses)

b. Caso haja dois máximos (mínimos) consecutivos, seleciona-se o maior (menor). 
c. Eliminam-se máximos e mínimos de forma que a distância entre dois máximos (mínimos), seja maior ou igual a 15 meses.

4. Computa-se o MCD (months of cyclical dominance) da série:

a. Considera-se a Curva de Spencer da série como sendo sua parte regular (tendência + ciclo).

b. Determina-se a parte irregular, It, tomando a diferença entre a série original e a Curva de Spencer: $I_{\mathrm{t}}=x_{t}-S_{t} x$.

c. Calcula-se $\operatorname{MCD}(\mathrm{j})=$, para $\mathrm{j}-1,2 . .6$.

d. MCD será o menor j para o qual $\mathrm{MCD}(\mathrm{j})$ é menor que a unidade.

5. Os pontos de inflexão determinados no passo anterior são refinados usando a série de media móvel de MCD meses:

a. Para cada máximo (mínimo) determinado no passo III, seleciona-se a maior (menor) observação na série de media móvel que dele diste até 5 observações.

b. Caso haja dois máximos (mínimo) consecutivos, seleciona-se o maior (menor).

6. Identifica-se, para cada máximo (mínimo) determinado no passo anterior, a maior (menor) observação na série que dele diste até Max (4, MCD) observações:

a. Caso haja dois máximos (mínimos) consecutivos, seleciona-se o maior (menor).

b. Eliminam-se os pontos de inflexão que estejam até seis meses do início ou fim da série.

c. Eliminam-se máximos e mínimos de forma que a distância mínima entre dois máximos (mínimos) consecutivos seja maior ou igual a 15 meses.

d. Eliminam-se máximos e mínimos de forma que a distância mínima entre um máximo e um mínimo consecutivos, e vice-versa, seja maior ou igual a seis meses. 


\section{APÊNDICE A3 - DATAÇÃO: INDICADOR DE STOCK-WATSON}

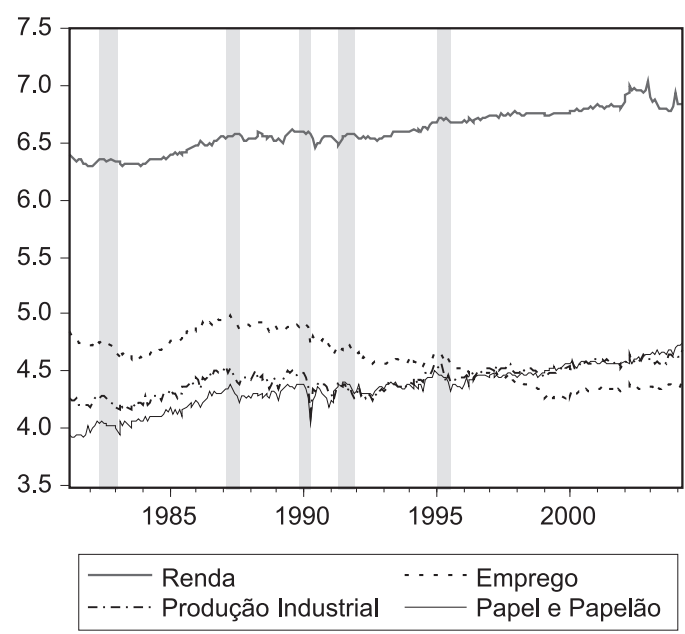

APÊNCIDE A4 - DATAÇÃO: INDICADOR DE MARIANO-MURASAWA

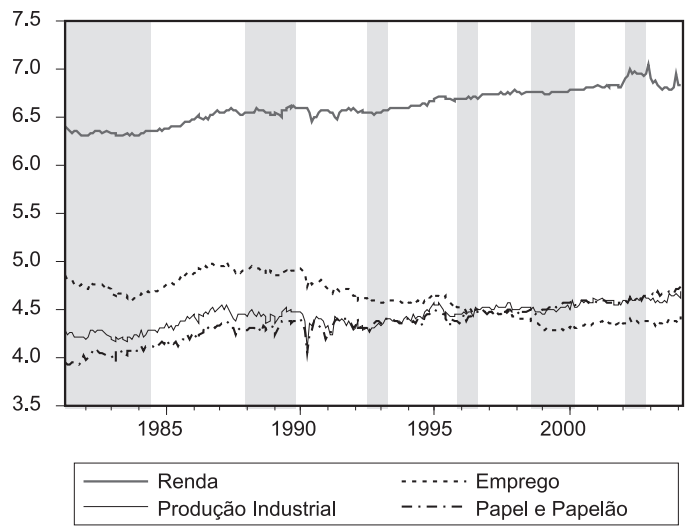




\section{APÊNDICE A5}

No primeiro gráfico, temos a datação utilizando o método do TCB modificado. No segundo gráfico, a datação do TCB padrão.
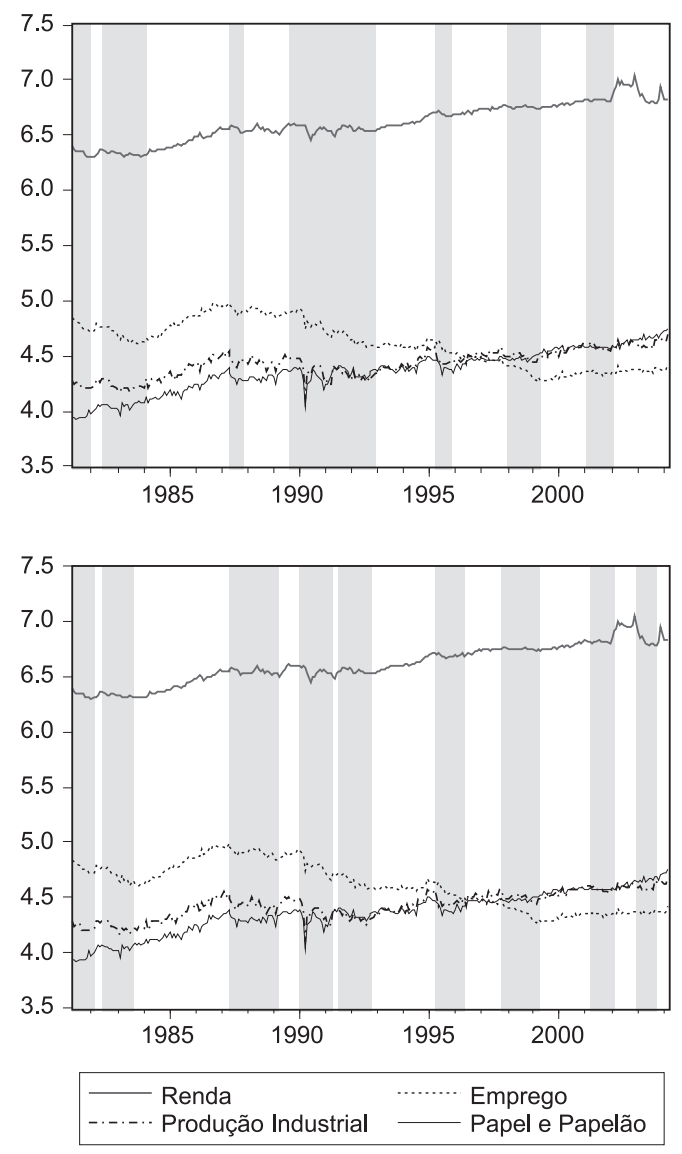